\title{
Single Pulmonary Nodule Management: Experience of a Moroccan Center about 24 Patients
}

\author{
Harmouchi Hicham ${ }^{1}$, Rabiou Sani ${ }^{1}$, Sidibe Kassim², Issoufou Ibrahim1, Belliraj Layla1, \\ Ammor Fatimazahra1, Lakranbi Marouane', Ouadnouni Yassine ${ }^{1,3}$, Smahi Mohammed 1,3 \\ ${ }^{1}$ Department of Thoracic Surgery, CHU Hassan II of Fez-Morocco, Fez, Morocco \\ ${ }^{2}$ Department of Imagery, CHU Hassan II of Fez-Morocco, Fez, Morocco \\ ${ }^{3}$ Faculty of Medicine and Pharmacy, Sidi Mohamed Ben Abdellah University, Fez, Morocco \\ Email: ^harmouchi.hicham@gmail.com
}

How to cite this paper: Hicham, H., Sani, R., Kassim, S., Ibrahim, I., Layla, B., Fatimazahra, A., Marouane, L., Yassine, O. and Mohammed, S. (2018) Single Pulmonary Nodule Management: Experience of a Moroccan Center about 24 Patients. Open Journal of Thoracic Surgery, 8, 43-49. https://doi.org/10.4236/ojts.2018.82008

Received: May 10, 2018

Accepted: June 23, 2018

Published: June 27, 2018

Copyright $\odot 2018$ by authors and Scientific Research Publishing Inc. This work is licensed under the Creative Commons Attribution International License (CC BY 4.0).

http://creativecommons.org/licenses/by/4.0/

(C) (i) Open Access

\begin{abstract}
Introduction: The management of single pulmonary nodule remains difficult, since it is of multiple etiologies. The endemicity of tuberculosis and hydatidosis in our context makes the etiological orientation even more difficult. Materials and Methods: This is a retrospective study, involving 24 patients, all operated on for a single pulmonary nodule in the department of thoracic surgery of CHU Hassan II of Fez over a period of 8 years. Results: There were 15 men and 9 women, with an average age of 51 years. $45.4 \%$ of patients had a history of known neoplasia. Discovery was fortuitous in $54 \%$ of cases. The radiologic diagnosis based on thoracic computed tomography had objectified an isolated nodule in all the patients with a right localization in $71 \%$ of the cases It was a solid pulmonary nodule in $91.5 \%$ of cases, and a frosted glass appearance in $8.5 \%$. Bronchial fibroscopy was contributory in one case which was a carcinoid tumor. The approach was a conservative postero-lateral thoracotomy in 22 patients. The gesture was atypical resection in $67 \%$, and lobectomy in $24.5 \%$ of cases, in the context of centrilobular nodules. The etiologies were dominated by pulmonary metastases in $29 \%$ of cases, pulmonary tuberculosis in $21 \%$ of cases. The postoperative course was simple in all patients. Conclusion: The pathological antecedents and the characteristics of the nodule on the imaging are essential elements allowing an etiological orientation. However, the pathological study remains the only method to confirm the diagnosis.
\end{abstract}

\section{Keywords}

Pulmonary Nodule, Computed Tomography, Diagnostic, Metastasis,

Tuberculoma, Surgery 


\section{Introduction}

The pulmonary nodule is defined as round or oval opacity surrounded by pulmonary parenchyma and measuring between $3 \mathrm{~mm}$ and $3 \mathrm{~cm}$ [1]. Etiologies are multiple, and the principle of management is not to ignore a malignant nodule at a curable stage, and conversely not to perform invasive explorations for a benign nodule [2]. The fortuitous discovery is frequent; the clinical and radiological context makes it possible to guide the diagnosis. The use of a surgical procedure remains for diagnostic purposes by excisional nodule biopsy, or curative pulmonary resection adjusted in case of non-metastatic malignant nodule. The objective of this series was to highlight the etiological profile, while relating our experience in the management of pulmonary nodules with diagnostic and therapeutic means that remain limited.

\section{Materials and Methods}

This was a retrospective study performed in the department of thoracic surgery of the CHU Hassan II of Fez, concerning 24 patients, selected between January 2010 and December 2017, who were treated for single pulmonary nodule. We have excluded any pulmonary nodule that has been associated with another homolateral or contralateral nodule, or a subpleural nodule, or a pulmonary mass, or mediastinal adenopathies or pleural effusion. Epidemiological, clinical and therapeutic data and histological diagnosis were recorded in a previously established form. The data analysis was done using the Excel PAC Office 2016 software for Windows.

\section{Results}

There were 24 patients ( 15 men and 9 women), with a sex ratio of 1.6 (Table 1 ). The median age was 51 years old. Of these patients, $45.4 \%$ had a history of known neoplasia. The notion of chronic active smoking was noted in 6 patients (25\%). Three (12.5\%) patients had been treated for pulmonary TB and declared cured. The finding was fortuitous in 54\% of cases (13 patients), including 11 as part of an extension of known neoplasia, and 2 following closed chest trauma (Figure 1). In 1 case, it was a nodule discovered on a chest CT scan following the assessment of a secondary spontaneous pneumothorax. The warning signs were cough in $27 \%$ of cases, hemoptysis in $17 \%$ of cases, and chest pain in $2 \%$ of cases. Chest-based computed tomography (CT)-based CT imaging showed 17 patients (71\%) with right lung lesions and 7 patients with left lung (29\%). Densitometric and volumetric analysis made it possible to note the solid character of the pulmonary nodule in 22 patients $(91.5 \%)$ with an average size of $2 \mathrm{~cm}$; in the other 2 patients (8.5\%), it was a frosted glass appearance with an average height of 1.7 $\mathrm{cm}$. Bronchoscopic bronchoscopy performed in 7 patients (29\%), had been contributory in a single case in a patient who had a carcinoid tumor. In a patient with a peripheral nodule, a lung biopsy made by CT scan was negative. Given the absence of PET-Scan in our context, the decision of a surgical biopsy was re- 
tained for all patients. This was a diagnostic and therapeutic procedure and the approach was a conservative postero-lateral thoracotomy in 22 patients. The indication of a video-assisted thoracic surgery concerned only 2 patients. The surgical procedure consisted of excisional biopsy in 67\% of cases, lobectomy in $24.5 \%$ of cases, and peri-kystectomy in $8.5 \%$ of cases. Diagnostic confirmation of the pulmonary nodule was obtained after the extemporaneous examination in 7 patients (29\%). For the other patients the diagnosis was obtained after the final histological and immunohistochemical examination with a variable delay between 7 and 17 days. It was a lung metastasis of a known cancer in $29 \%$ of cases, pulmonary tuberculosis in its nodular form in $21 \%$ of cases, primary lung cancer in $12.5 \%$ of cases and 'a benign lung tumor in $12.5 \%$ of cases. In $8.5 \%$ of cases it was a carcinoid tumor and a pulmonary hydatid cyst in $8.5 \%$ of cases. We also noted an aspergilloma and a pseudo-inflammatory lung tumor in $4 \%$ for each (Table 2). The follow-up was simple in all patients, with an average hospital stay of 5 days. No case of death related to the gesture was recorded during the thirty days postoperatively. With a mean follow-up of 2 years we have not noted a recurrence to date. Patients who had a metastatic nodule of a known neoplasm, or a malignant nodule were referred to the oncology department for further management after a discussion in a multidisciplinary consultation meeting. Those who had an active form of tuberculosis were referred to the specialized diagnostic center. While some patients required clinical and radiological monitoring.

Table 1. Characteristics of patients.

\begin{tabular}{lc}
\hline & Number of patients (percentage) \\
\hline Total & $24(100 \%)$ \\
Sex & $15(62.5 \%)$ \\
-Men & $9(37.5 \%)$ \\
-Women & 51 \\
Median age & \\
Pathological history & $11(45.4 \%)$ \\
-History of known neoplasia & $6(25)$ \\
-Chronic active smoking & $3(12.5)$ \\
-History of pulmonary TB & $13(54 \%)$ \\
Fortuitous discovery & $11(46 \%)$ \\
-imaging of known neoplasia & $2(8 \%)$ \\
-chest trauma & $11(46 \%)$ \\
Clinical sign & $6(27 \%)$ \\
-cough & $4(17 \%)$ \\
-hemoptysis & $1(2 \%)$ \\
-chest pain & $17(71 \%)$ \\
- Right side & $7(29 \%)$ \\
-Left side & $22(92 \%)$ \\
solid character of nodule & $2(8 \%)$ \\
ground-glass opacity &
\end{tabular}


Table 2. Etiologies of pulmonary nodules.

\begin{tabular}{lc}
\hline Etiologies of pulmonary nodules & Percentage \% \\
\hline Pulmonary metastases & $29 \%$ \\
○ Digestive origin & $(12.5 \%)$ \\
○ Uterine origin & $(8.5 \%)$ \\
○ Pulmonary origin & $(4 \%)$ \\
○ Breast origin & $4 \%$ \\
Tuberculoma: & $(21 \%)$ \\
Primary pulmonary tumor & $12.5 \%$ \\
○ Adenocarcinoma & $(8.5 \%)$ \\
○ Squamous cell carcinoma & $(4 \%)$ \\
Benign lung tumors & $12.5 \%$ \\
O Hamartochondrome & $(8.5 \%)$ \\
○ Hamartoma & $(4 \%)$ \\
Carcinoid tumors & $(8.5 \%)$ \\
Hydatid cyst & $(8.5 \%)$ \\
$\quad$ Aspergilloma & $(4 \%)$ \\
Inflammatory pseudo-tumoral nodule & $(4 \%)$ \\
\hline
\end{tabular}

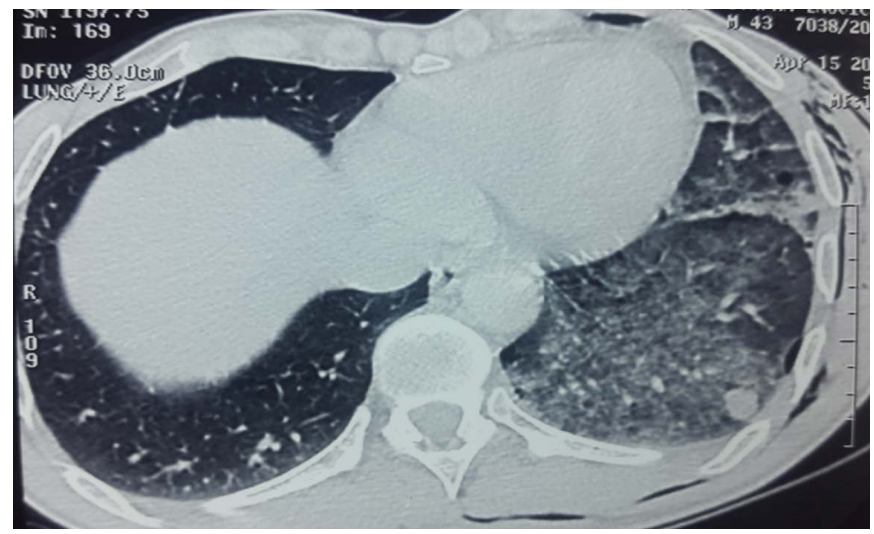

Figure 1. Fortuitous finding of a solid pulmonary nodule of the left lower lobe in a patient who was admitted to the emergency department for closed chest trauma, with foci of pulmonary contusion and subcutaneous emphysema.

\section{Discussion}

The pulmonary nodule is defined as any focal opacity of the pulmonary parenchyma measuring between $3 \mathrm{~mm}$ and $3 \mathrm{~cm}$. It can be solid when it completely erases the structures of the underlying parenchyma, or be in frosted glass if certain vascular or aerial pulmonary elements remain visible, whereas the mixed nodule consists of a solid component and frosted glass at the same time [3] [4]. The key to diagnostic management is not to neglect a malignant nodule at a curable stage, but also to reduce the iatrogenic risk and not perform invasive explorations for a benign nodule [4]. The frequency of solid nodules in our study can be explained by the frequency of metastatic nodules and those of tubercu- 
lous origin, whose expression is generally in solid form. The fortuitous discovery of pulmonary nodules is frequent, given the increased use of thoracic CT, and the rarity of pulmonary nodule pulmonary symptomatology [5]. The average size of the nodules in our series explains their weak detection on the chest X-ray, since the sensitivity does not exceed $50 \%$ below a size of $2 \mathrm{~cm} \mathrm{[6].} \mathrm{Thoracic} \mathrm{CT}$ allows a precise analysis of the nodule, objectifying its size, its contours, and its content (presence of fat, air, or calcifications). It also makes it possible to specify whether the nodule is solid, in frosted or mixed glass, which allows a first etiological orientation before considering a method of anatomopathological diagnosis. Unfortunately, no positron emission tomography (PET) was done in this study because of the high cost of the examination and its unavailability in our university hospital. This test has a sensitivity of $95 \%$ and a specificity of $80 \%$ to differentiate between a benign and malignant nodule [7]. Bronchial fibroscopy, which reports the diagnosis of proximal carcinoid tumor in a single patient, is of low cost for the peripheral locations of pulmonary nodules [8]. Etiologies of pulmonary nodules are multiple, and are determined according to whether the nodule is solid, frosted glass or mixed. Les nodules solides sont d'origine tumorale maligne dans une minorité des cas (Figure 2), Solid nodules are of malignant tumor origin in a minority of cases (Figure 2), however nodules in ground glass are most often of adenocarcinomatous origin at different stages of evolution [9] [10]. The context of endemicity in our country, either of tuberculosis or of hydatidosis, is to be taken into consideration. Tuberculosis has the nodular form of tuberculosis (Figure 3), and aspergilloma, whose primary etiology remains tuberculosis in our context, can also be expressed in nodular form. All our patients whose diagnostic orientation was a probably malignant nodule were the subject of a discussion at a multidisciplinary consultation meeting. Pulmonary nodule surgery is indicated for either diagnostic or therapeutic purposes. It is done either by a thoracotomy or video-assisted thoracic surgery [4]. When the surgeon is confident of a diagnosis of malignancy preoperatively by obtaining a positive endoscopic or transthoracic biopsy, radical excision

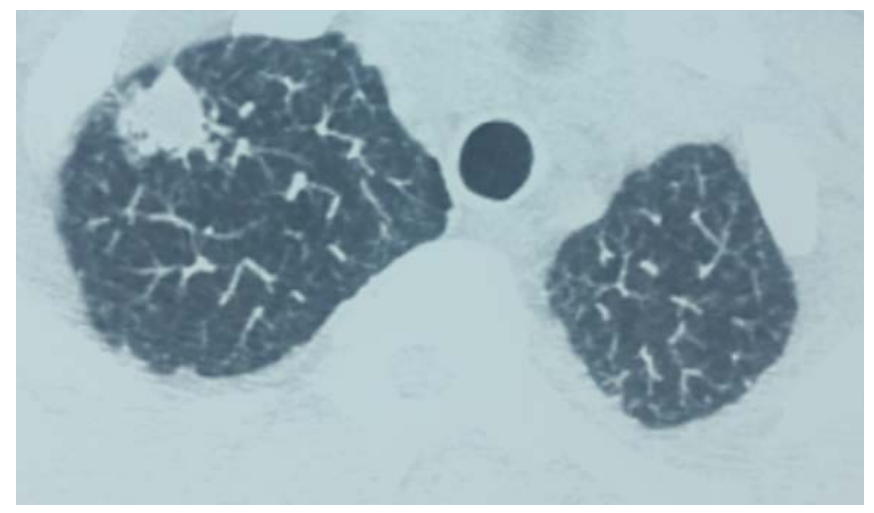

Figure 2. Peripheral solid pulmonary nodule of the right upper lobe, irregular contours, in a former chronic smoker, whose pathological study was a pulmonary adenocarcinoma, benefiting from a right superior lobectomy with lymph node dissection. 


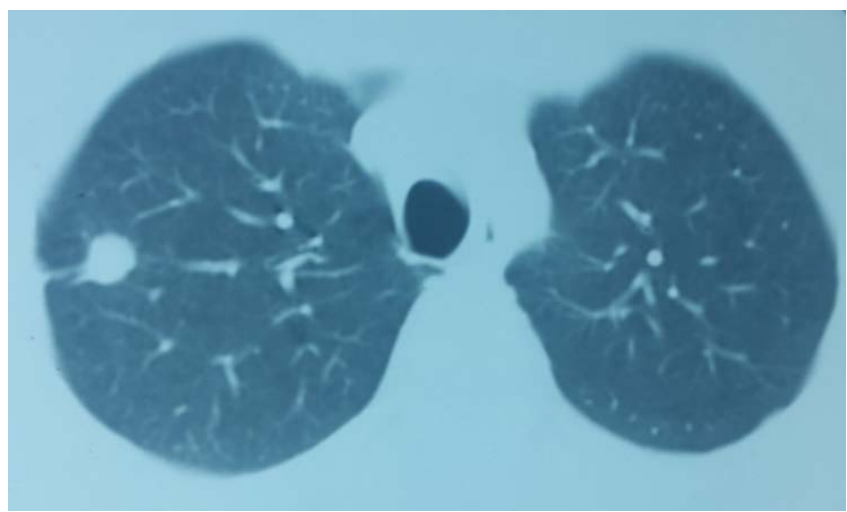

Figure 3. solid pulmonary nodule of the right upper lobe, with regular contours, in a former tuberculous patient, whose pathological study was a tuberculoma.

is performed without extemporaneous histological control of the tumor [11]. Intra-operative exploration may in some cases have the diagnosis as in the case of hydatid cyst or tuberculoma, as well as hamartochondromas whose palpation finds a cartilaginous component. However, in doubtful cases, an extemporaneous examination is desirable to have the diagnosis and complete the gesture by a controlled excision in the case of non-metastatic malignant nodules, or to stop at an excisional nodule biopsy. Video-assisted thoracic surgery has fewer complications than open surgery, and shorter hospital stay. However, this technique was only performed in two patients in this study.

\section{Conclusion}

The management of the single pulmonary nodule remains difficult, since it is of multiple etiologies, and the non-easy use of certain diagnostic means. Surgery of the pulmonary nodule is either for diagnostic purposes, especially for nodules with a known neoplasm context, or therapeutic in the case of non-metastatic malignant nodules.

\section{References}

[1] Revel, M.-P. (2014) Diagnosis of Solitary Pulmonary Nodule. Feuillets de Radiologie, 1-20.

[2] Revel, M.-P. (2012) Diagnosis of Solitary Pulmonary Nodule. EMC - Radiologie et Imagerie Médicale - Cardiovasculaire - Thoracique - Cervicale, 7, 32, 366-A-10.

[3] Tuddenham, W.J. (1984) Glossary of Terms for Thoracic Radiology: Recommendations of the Nomenclature Committee of the Fleischner Society. AJR. American Journal of Roentgenology, 143, 509-17. https://doi.org/10.2214/ajr.143.3.509

[4] Westeel, V., Pernet, D. and Chapuy, S. (2014) Which Management for Pulmonary Nodule ? Revue des Maladies Respiratoires Actualités, 6, 373-378. https://doi.org/10.1016/S1877-1203(14)70591-9

[5] Laurent, F. and Rémy, J. (2002) Management Strategy for Pulmonary Nodules. Revue des Maladies Respiratoires, 19, 715-725.

[6] De Boo, D.W., Uffmann, M., Bipat, S., Boorsma, E.F., Scheerder, M.J., Weber, M., et 
al. (2010) Gray-Scale Reversal for the Detection of Pulmonary Nodules on a PACS workstation. AJR. American Journal of Roentgenology, 197, 1096-1100.

https://doi.org/10.2214/AJR.11.6625

[7] Groheux, D., Hindie, E., Tredaniel, J., Giraudet, A.L., Vaylet, F., Berenger, N., et al. (2009) PET-CT for Evaluation of the Solitary Pulmonary Nodule: An Update. Revue des Maladies Respiratoires, 26, 1041-1055. https://doi.org/10.1016/S0761-8425(09)73531-4

[8] Popovitch, T., Kvale, P.A. and Eichenhorn, M.S. (1982) Diagnostic Accuracy of Multiple Biopsies from flexible Fiberoptic Bronchoscopy: A Comparison of Central versus Peripheral Carcinoma. The American Review of Respiratory Disease, 125, 521-523. https://doi.org/10.1164/arrd.1982.125.5.521

[9] Ruppert, A.M., Lerolle, U., Carette, M.F., Lavole, A., Khalil, A., Bazelly, B., et al. (2010) Coexisting Pulmonary Nodules in Operable Lung Cancer: Prevalence and Probability of Malignancy. Lung Cancer, 74, 233-238.

https://doi.org/10.1016/j.lungcan.2011.03.012

[10] Kim, H.Y., Shim, Y.M., Lee, K.S., Han, J., Yi, C.A. and Kim, Y.K. (2007) Persistent Pulmonary Nodular Ground-Glass Opacity at Thin-Section CT: Histopathologic Comparisons. Radiology, 245, 267-275. https://doi.org/10.1148/radiol.2451061682

[11] Moliniéa, V., Duchatelle, V., Abbey-Tobby, A. and Balaton, A. (2012) Extemporaneous Examination in Lung Pathology: The Pathologist's View. Revue des Maladies Respiratoires, 29, 836-843. 